\title{
ÉTUDE DU RAPPORT ENTRE \\ L'ÉCLAIREMENT RELATIF SOUS FORÊT \\ ET L'ÉGLAIREMENT LUMINEUX INGIDENT \\ EN PHÉNOPHASE FEUILLÉE (*)
}

par

G. Vyncke

1. Introduction

O.D.C. $181.21--015$

Une juste appréciation de l'éclairement lumineux transmis sous le couvert forestier est d'une grande importance, surtout par rapport à l'étude du microclimat dans les groupes de régénération. Pour caractériser les conditions lumineuses sous forêt, on recourt souvent aux valeurs d'éclairement relatif. Ainsi l'éclairement transmis sous forêt est exprimé en pour-cent de l'éclairement lumineux incident (au-dessus des cimes ou en plein découvert). Ces valeurs relatives peuvent être calculées d'après des observations instantanées et synchroniques, d'après des données périodiques fournies par des appareils totalisateurs ou enregistreurs et d'après des lectures de diagrammes fournis par les enregistreurs pour des moments correspondants. En outre, il est possible selon l'objet de recherches spécifiques, de mesurer l'éclairement relatif lumineux sous forêt directement à l'aide d'appareils spéciaux (Brechtel 1962, Vyncke et Goossens 1968).

Dans les publications concernant les conditions lumineuses sous forêt, différents auteurs (Geiger 1961, Sa u berer et Ha er tel 1959, Mitscherlich et al. 1965, 1966, 1967) ont constaté que l'éclairement relatif n'est pas stable pour une place déterminée, mais que sa valeur relative dépend de l'éclairement lumineux incident,

Cette dépendance observée sous des dispositifs d'ombrage installés artificiellement au-dessus des plantations de jeunes hêtres, a fait l'objet d'une étude par Burschel et Schmaltz (1965). Ils ont établi, dans chacun des cinq degrés d'ombrage, une dé-

(*) Programme du Centre National d'Ecologie Générale (Bruxelles) subventionné par le Fonds de la Recherche fondamentale collective. 
croissance hyperbolique de l'éclairement relatif avec l'accroissement de l'éclairement absolu en plein découvert.

Dans les stations écologiques du Centre National d'Ecologie Générale (G.N.E.G.) à Virelles, de nombreuses mesures de lumière ont été effectuées au cours des années 1965-66 à l'aide d'apparcils totalisateurs et enregistreurs.

$\mathrm{Vu}$ la faible valeur de lumière visible atteignant le sol sous le couvert feuillé et le grand nombre de données disponibles pour la phénophase feuilléc fournies par différents appareils, il serait intéressant de contrôler comment se présente la relation citée. Le cours de l'éclairement relatif à l'accroissement de l'éclairement lumineux incident pour les conditions sous forêt est-il calculable?

En même temps se pose la question suivante : De quelle façon l'éclairement relatif dans la phénophase feuillée varie-t-il au cours d'une journée?

\section{Méthodologie}

\subsection{Description de la parcelle étudiée}

Les mesures ont été effectuées dans les stations écologiques de Virelles (lat. nord : $50^{\circ} 03^{\prime} 23^{\prime \prime}$; long. est : $4^{\circ} 20^{\prime} 20^{\prime \prime}$; alt. $245 \mathrm{~m}$ ).

Les éclairements sous forêt ont été mesurés dans une chênaie mélangée calcicole (Virelles-Blaimont). La jeune futaie est issue d'une conversion d'un taillis-sous-futaie et est composée de Quercus robur, Carpinus betulus, Acer campestre et Fraxinus excelsior, ainsi que d'autres essences secondaires.

Le tableau 1 présente un aperçu schématique des données principales de l'inventaire forestier.

TABLEAU I

Inventaire de la parcelle étudiée

\begin{tabular}{|l|c|c|c|c|c|}
\hline \multicolumn{1}{|c|}{ Essences } & $\begin{array}{c}\text { Quercus } \\
\text { robur }\end{array}$ & $\begin{array}{c}\text { Carpinus } \\
\text { betulus }\end{array}$ & $\begin{array}{c}\text { Acer } \\
\text { campestre }\end{array}$ & $\begin{array}{c}\text { Fraxinus } \\
\text { excelsior }\end{array}$ & $\begin{array}{c}\text { Total } \\
\text { par ha }\end{array}$ \\
\hline $\begin{array}{l}\text { Nombre de } \\
\text { tiges par ha }\end{array}$ & 220 & 420 & 80 & 80 & 800 \\
\hline $\begin{array}{l}\text { Composition } \\
\text { en pour-cent }\end{array}$ & 27,5 & 52,5 & 10 & 10 & 100 \\
\hline $\begin{array}{l}\text { Surface ter- } \\
\text { rière en } \mathrm{m}^{2} / \mathrm{ha}\end{array}$ & 17,31 & 4,15 & 1,18 & 2,66 & 25,30 \\
\hline $\begin{array}{l}\text { Volume } \\
\text { en } \mathrm{m}^{3} / \mathrm{ha}\end{array}$ & 184,432 & 29,084 & 9,462 & 24,673 & 247,651 \\
\hline
\end{tabular}


Un rajeunissement riche surtout en Fraxinus excelsior, Acer pseudoplatamus et Acer campestre, se rencontre sous un couvert relativement opaque. La voûte foliacée présente en effet une grande densité et la fermeture totale du massif se faisait au cours de l'année 1966. Cela se manifeste très clairement lors de l'étude des éclairements relatifs moyens pour les périodes feuillées de 1965 et 1966. Cette valeur moyenne s'élève à $3 \%$ environ en 1965 et à $1 \%$ en 1966.

Les mesures de l'éclairement lumineux incident ne sont pas notées au-dessus des cimes, mais ont lieu en plein découvert dans la station écoclimatologique sur prairie permanente (VirellesPrairie Philippart, alt. $245 \mathrm{~m}$ ).

\subsection{Description sommaire de l'appareillage}

Les observations de l'éclairement lumineux ont été effectuées à l'aide de cellules photoélectriques au sélénium du type Lange S50. Les cellules sont sensibles au rayonnement visible entre les longueurs d'onde de $370 \mathrm{~nm}$ à $780 \mathrm{~nm}\left({ }^{*}\right)$. Elles présentent un certain désaavantage quant à leur fonction sélective dans leur portée spectrale, intéressante pour l'énergie photosynthétique. Par contre, leur courbe de sensibilité et la courbe de sensibilité de l'œil humain coöncident. L'une et l'autre ont un maximum situé à environ $550 \mathrm{~nm}$

L'utilisation des cellules au sélénium dans et hors forêt implique l'utilisation de filtres de densité neutre (filtres d'opale Schott-Jena), parce qu'une relation linéaire entre le flux photoélectrique et l'éclairement n'existe qu'au-dessous de 1000 lux. Par conséquent, les éclairements à mesurer doivent être réduits aux valeurs inférieures à I000 lux.

Les cellules photoélectriques au sélénium du type Lange sont souvent employées en Europe en vie d'observations des éclairements lumineux dans les forêts et pour des recherches analogues (Brechtel 1962; Van Miegroet 1963, 1965; Mitscherlich et al. $1965,1966,1967)$.

Les cellules sont connectées aux appareils totalisateurs et enregistreurs. Ceux-ci mesurent le flux lumineux. Les appareils totalisateurs indiquent au moyen d'un compteur la quantité de potentiel électrique engendrée par les cellules pour une période bien déterminée.

Ils sont étalonnés individuellement et ont comme unité la lux-heure.

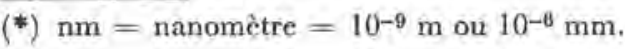


Les appareils enregistreurs marquent l'ampérage engendré successivement par trois cellules différentes. Toutes les 20 secondes, l'aiguille frappe en notant sur le papier un point coloré.

L'étalonnage de l'ampérage fourni par les cellules a été réalisé en laboratoire au moyen d'un filtre d'interférence monochromatique (Schott-Jena), qui sélectionne une bande spectrale centrée sur le maximum de la courbe de sensibilité de la cellule. Un appareil Weston étalonné en lux a été employé comme référence. L'étalonnage de l'ampérage a été possible par l'intermédiaire des potentiomètres réglables.

\subsection{Aperçu de l'installation et particularités}

Pour la saison 1965 les mesures de l'éclairement lumineux ont été réalisées pendant les mois de juillet, d'août et de septembre. A ceux-ci s'ajoute le mois de juin pour la saison 1966. Les appareils totalisateurs étaient lus chaque semaine, ainsi que les valeurs de l'éclairement lumineux représentant une valeur hebdomadaire. Les mesures au moyen des enregistreurs n'ont été effectuées que deux ou trois jours par semaine, étant donné l'impossibilité pratique d'enregistrement quotidien. Les courbes enregistrées ont été planimétrées. Pour chaque journée la quantité lux-heure a été établie en tenant compte des facteurs réducteurs, comme celui du filtre de densité neutre, le facteur du shunt employé et le facteur de l'étalonnage.

La longueur de la journée enregistrée étant connue, il est possible de calculer la valeur moyenne de l'éclairement lumineux journalier d'une manière évidente.

Dans la parcelle de référence (Prairie Philippart) deux appareils enregistreurs (6 cellules) et quatre totalisateurs ont été placés. Sous forêt (Bois de Blaimont): quatre enregistreurs (12 cellules) et neuf totalisateurs.

Les cellules ont été disposées à $1 \mathrm{~m}$ du sol et sont divisées sur une surface clôturée de $10 \mathrm{~m}$ sur $10 \mathrm{~m}$ d'une façon régulière.

\section{Rapport entre l'éclairement relatif sous forêt et l'éclairement incident}

En partant des éclairements relatifs moyens hebdomadaires à l'égard des mesures de référence absolues en plein découvert (appareils totalisateurs), les coefficient de corrélation entre l'éclairement relatif sous forêt et l'éclairement incident n'est pas significatif, ni pour les mois de 1965 ni pour ceux de 1966.

Néanmoins, à première vue, les grandes valeurs relatives peuvent être reliées aux intensités les plus basses, ainsi que les 
pour-cents bas aux intensités les plus grandes. La même remarque peut être formulée pour les données fournies par les appareils enregistreurs. C'est-à-dire qu'entre l'éclairement relatif sous forêt, calculé des éclairements lumineux incidents et transmis sous forêt, et l'éclairement incident il n'existe pas une corrélation significative.

Pour la validité de l'explication, il faut dire qu'il s'agit de valeurs hebdomadaires ou journalières de l'éclairement lumineux, qui sont donc représentatives pour des périodes bien déterminées (une semaine ou une journée) et dans lesquelles de grandes fluctuations d'intensité absolue sont présentés. Dans ce contexte, il est déjà signalé qu'une restriction doit être faite en matière du jugement de l'éclairement relatif calculé d'après des observations fournies par des appareils totalisateurs (Vyncke et Goossens 1968).

Au moyen de diagrammes journaliers enregistrés il a été facile de déduire l'éclairement absolu au midi vrai, de façon que 27 journées ont été disponibles pour la période d'observation 1965 et 32 journées pour 1966. A partir des diagrammes d'observation, les journées ont été classées sommairement selon le type de temps de la manière suivante (Grulois et Vyncke 1969):

$\mathrm{S}$ : ciel serein, nébulosité nulle au très faible.

SN : ciel en partie nuageux avec de longues éclaircies.

$\mathrm{N}$ : ciel nuagcux avec de courtes éclaircies durant toute la journée.

D : ciel couvert, très nuageux, aucune éclaircies.

Le tableau II présente un aperçu de la répartition des types de temps durant les périodes d'observation.

TABLEAU II

Répartition des types de temps

durant les périodes d'observation en 1965 et 1966

\begin{tabular}{|c|c|c|c|c|}
\hline \multirow{2}{*}{ Type de temps } & \multicolumn{2}{|c|}{1965} & \multicolumn{2}{|c|}{1966} \\
\hline & $\begin{array}{c}\text { nombre de } \\
\text { jours }\end{array}$ & $\%$ & $\begin{array}{c}\text { nombre de } \\
\text { jours }\end{array}$ & $\%$ \\
\hline $\begin{array}{c}\text { Jours sereins } \\
\text { (S) }\end{array}$ & 5 & 19 & 7 & 22 \\
\hline $\begin{array}{l}\text { Jours sercins-nuagcux } \\
\text { (SN) }\end{array}$ & 2 & 7 & 5 & 15 \\
\hline $\begin{array}{l}\text { Jours nuageux } \\
\text { (N) }\end{array}$ & 14 & 52 & 14 & 44 \\
\hline $\begin{array}{r}\text { Jours diffus } \\
\text { (D) }\end{array}$ & 6 & 22 & 6 & 19 \\
\hline Total & 27 & 100 & 32 & 100 \\
\hline
\end{tabular}


Pourtant seulement quelques jours, aussi bien en 1965 qu'en 1966 ont été retenus pour les recherches suivantes, parce que l'intensité absolue au moment du midi vrai n'a pu être toujours déterminée graphiquement d'une manière précise. C'est le cas notamment pour les jours sereins-nuageux (SN) et les jours nuageux $(\mathrm{N})$, de façon que 21 jours ont été retenus pour la période d'observation en 1965, et 21 jours pour la période en 1966 .

Entre l'éclairement relatif sous forêt et l'éclairement absolu incident, à partir des intensités lumineuses au midi vrai, il existe une relation mathématique. Ceci est démontré par le tableau III, dans lequel sont notés les droites de régression et les coefficients de corrélation correspondants avec leurs valeur de t respectives pour les périodes d'observation.

\section{TABLEAU III}

Droites de régression, coefficients de corrélation et la signification de la valeur de $t$ correspondante pour les périodes d'observation $(\mathrm{x}=\mathrm{l}$ 'ćclaircment lumineux incident en Klux

$y=$ l'éclairement relatif en pour-cent

\begin{tabular}{|c|c|c|c|}
\hline $\begin{array}{c}\text { Période } \\
\text { d'observation }\end{array}$ & $\begin{array}{c}\text { Droite de } \\
\text { régression }\end{array}$ & $\begin{array}{c}\text { Coeflicient de } \\
\text { corrélation }\end{array}$ & Valeur de $t$ \\
\hline 1965 & $y=3,6664-0,0148 x$ & $r=-0,6969$ & $t=4,2353000$ \\
1966 & $y=1,3959-0,0057 x$ & $r=-0,7303$ & $t=4,6599000$ \\
\hline
\end{tabular}

La figure 1 présente les deux équations du premier degré du tableau III. La présentation graphique fait observer que le coefficient de régression pour $1965(-0,0148)$ est plus petit que celui pour $1966(-0,0057)$ ou en d'autres termes, que l'angle d'inclinaison de la droite est plus grand en 1965 qu'en 1966. En même temps, le niveau d'ombrage ou l'indíce de densité du couvert forestier pour les périodes d'observation feuillées est plus bas en 1966 qu'en 1965 ( $1 \%$ par rapport à $3 \%$ ), à raison de la fermeture totale des couronnes en 1966.

D'après la figure 1 et les deux coefficients de régression des droites peut être constaté que pour un changement identique de l'éclairement incident (en Klux), la transmission relative (en pourcent) devient plus petite selon que la fermeture du couvert feuillé se réalise ou que le niveau moyen d'ombrage devient plus petit.

En effet, l'éclairement relatif sous forêt est une fonction de l'éclairement absolu incident. La valeur relative est grande pour 


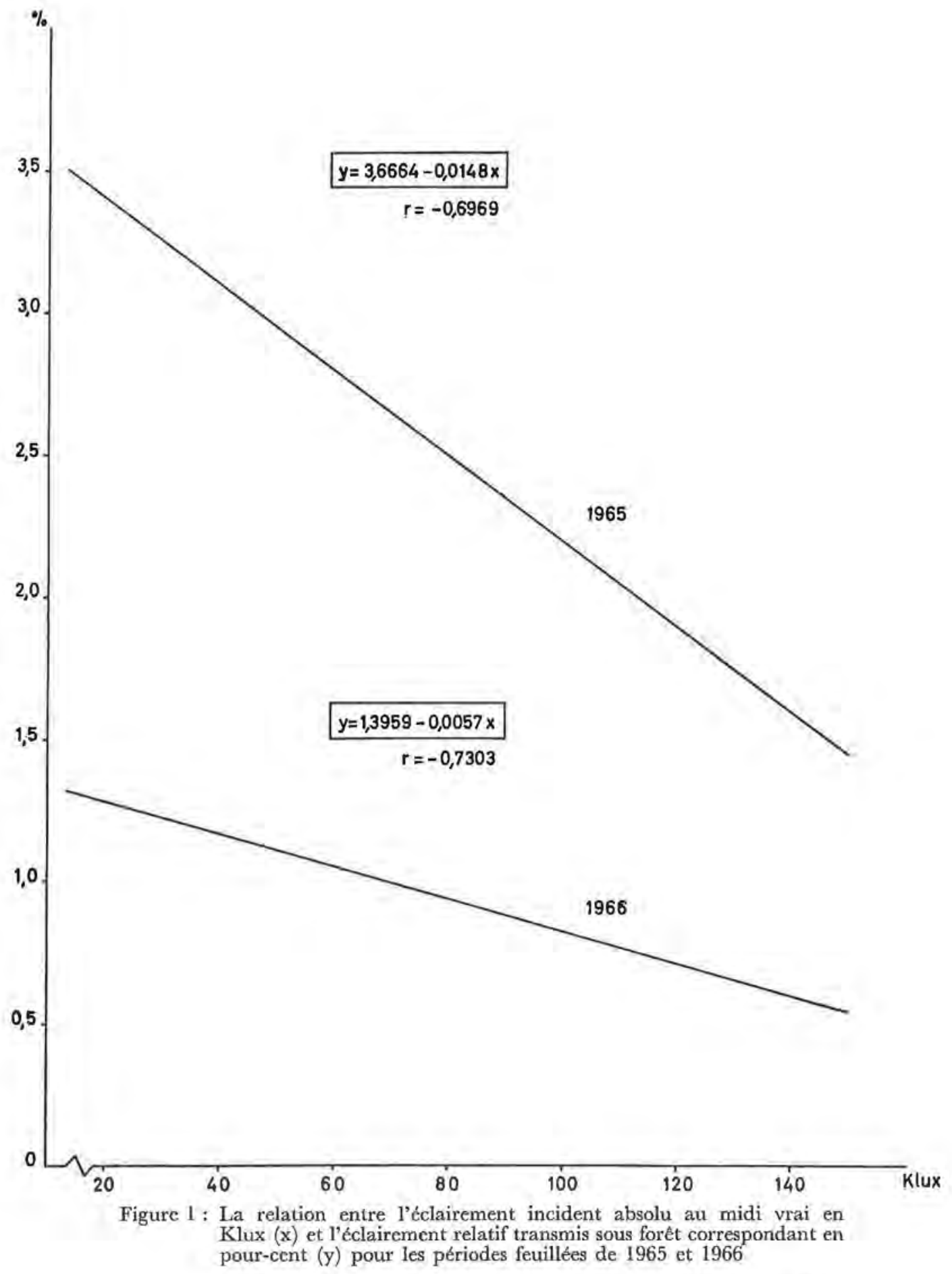


les intensités absolues basses et elle diminue avec l'accroissement de l'intensité absolue.

De plus, les intensités absolues basses au midi vrai sont notées pour les journées du type de temps diffus (D). Les grandes intensités sont observées, par contre, en des jours sereins (S).

A cette typologie les observations suivantes peuvent être ajoutées : pour les jours de type $\mathrm{S}$ la lumière au midi vrai est essentiellement du genre direct, tandis que pour les jours du type $\mathrm{D}$ le genre est avant tout indirect ou diffus. Le rapport trouvé permet de conclure que la lumic̀rc directe (les grandes intensités) atteint le sol herbacé sous couvert forestier relativement moins que la lumière diffuse (les basses intensités). En d'autres termes, la lumière directe est relativement plus absorbée et/ou plus réfléchie par le dôme de verdure que la lumière diffuse. Qu'elle soit plus absorbée est invraisemblable.

\section{Le cours de l'éclairement relatif pendant la journée}

Etant donné que l'éclairement lumineux change pendant la journée, la question se pose comment l'éclairement relatif évolue. Pour réaliser cette recherche, une journée de type serein (S) a été choisie. Comme pour ce type de temps la nébulosité est nulle ou très faible, le cours de l'intensité absolue est très stable, sans fluctuations intenses dues au changement de nébulosité. Les diagrammes d'enregistrements sous le couvert forestier et en plein découvert sont ainsi le mieux comparables pour la même journée aux différents moments. En même temps, le cours périodique de l'intensité enregistrée pour les jours sereins peut être assez bien ajusté mathématiquement par une fonction continue $f(x)$. En chaque point $\mathrm{x}$ de l'intervalle (lever du solcil, coucher du soleil) cette fonction peut être développée en série trigonométrique de Fourier sous la forme suivante :

$f(x)=\frac{a_{0}}{2}+a_{1} \cos x+b_{1} \sin x+a_{2} \cos 2 x+b_{2} \sin 2 x+\ldots$ $\ldots+a_{n} \cos n x+b_{n} \sin n x+\ldots$

où les coefficients de Fourier $a_{n}$ et $b_{n}$ se calculent d'après les formules (2) et (3)

$$
\begin{array}{ll}
a_{n}=\frac{1}{\pi} \int_{0}^{2 \pi} f(x) \cdot \cos n x \cdot d x & (n=0,1,2, \ldots) \\
b_{n}=\frac{1}{\pi} \int_{0}^{2 \pi} f(x) \cdot \sin n x \cdot d x & (n=1,2, \ldots)
\end{array}
$$


Les diagrammes d'enregistrement de l'éclairement lumineux transmis sous forêt montrent la particularité suivante : les valeurs de l'éclairement sont toujours plus élevées pour la période avant le coucher du soleil que pour la période après la lever du soleil, alors que la hauteur du soleil reste la même.

Ceci s'explique par une particularité de la station de mesure de l'éclairement sous forêt. Etablie sur un versant orienté vers l'ouest, la station présente un obstacle sur l'horizon consitué par un relief du terrain situé à l'est du point de mesure. Celui-ci interdit pendant quelque temps après le lever du soleil toute réception de l'éclairement lumineux et réduit ainsi la densité du flux lumineux transmis au sol (Grulois et Vyncke 1969).

Les diagrammes enregistrés le 9 septembre 1966 ont été utilisés, Les intensités de la période matinale ont été légèrement modifiées, afin d'obtenir un cours symétrique autour du midi vrai. Le jour choisi doit donc être considéré comme une journée sereine et idéalisée.

A l'aide de l'analyse Fourier les coefficients $a_{n}(2)$ et $b_{n}(3)$ ont été déterminés dans le polynôme, développé en série trigonométrique de Fourier (1) jusqu'à trois harmoniques $(\mathrm{n}=3)$. Comme l'ajustement mathématique pour le jour serein et idealisé a paru satisfaisant, le calcul d'harmoniques suivantes n'a pas été nécessaire.

Le cours périodique de l'intensité absolue pendant le jour serein idéalisé (le 9 septembre 1966) peut être présenté par les fonction (4) et (5), valables respectivement pour le cours de l'éclairement incident en plein découvert $\left(\mathrm{Y}_{\mathrm{p}}\right)$ et pour l'éclairement transmis au sol sous forêt $\left(\mathrm{Y}_{\mathrm{b}}\right)$ :

$Y_{p}=\frac{108,436}{2}-55,184 \cos x+14,786 \sin x-1,250 \cos 2 x+$

$+0,721 \sin 2 x+0,988 \cos 3 x-0,988 \sin 3 x$

$\mathrm{Y}_{\mathrm{b}}=\frac{0,650}{2}-0,284 \cos \mathrm{x}+0,076 \sin \mathrm{x}-0,027 \cos 2 \mathrm{x}+$

$+0,015 \sin 2 x-0,004 \cos 3 x+0,004 \sin 3 x$

La figure 2 présente les fonction (4) et (5). La valeur Y s'exprime en unité Kilolux (Klux) avec une autre échelle pour les fonction $\mathrm{Y}_{\mathrm{p}}$ et $\mathrm{Y}_{\mathrm{b}}$.

L'intervalle fermé entre le lever du soleil $\left(5 \mathrm{~h} 07^{\prime}\right)$ et le coucher du soleil (18h11') est présenté sur l'axe t. La largeur correspond à la longueur du jour (13h04').

Le cours symétrique de la courbe d'intensité autour du midi vrai permet le calcul des valeurs relatives dans le demi-intervalle (lever du soleil, midi vrai). Pour certaines valeurs de $x=t_{n}$ $(1,2,3, \ldots)$ et avec $\Delta t$ constant, les intensités correspondantes de 


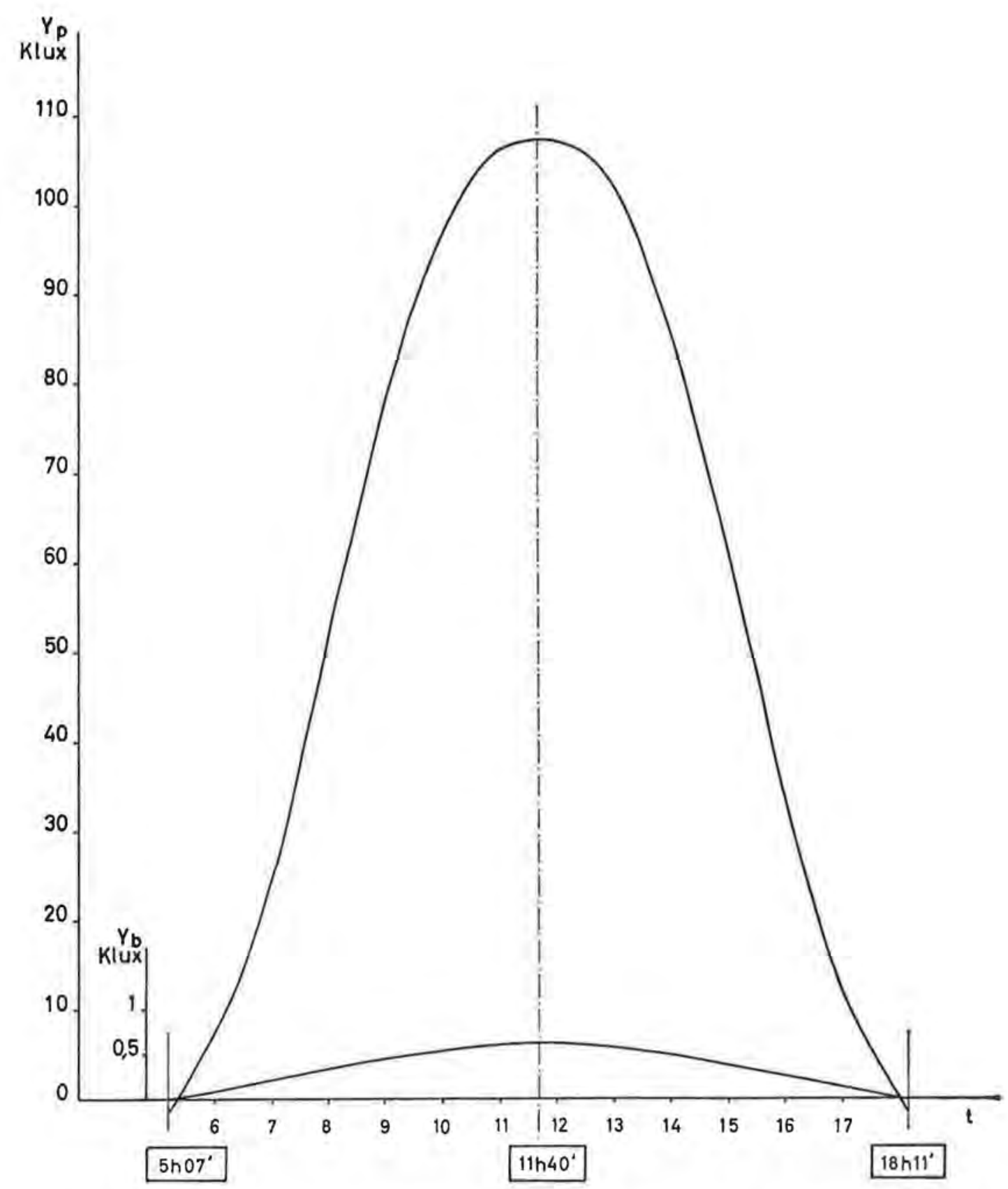

Figure 2: Le cours de l'éclairement incident $\left(\mathrm{Y}_{\mathrm{D}}\right)$ et transmis $\left(\mathrm{Y}_{\mathrm{b}}\right)$ sous forêt en Klux pendant un jour idéalisé du type de temps serein (le 9 septembre 1966) 
l'éclairement absolue $\mathrm{Y}_{\mathrm{p}}$ et $\mathrm{Y}_{\mathrm{b}}$ (en Klux) sont déterminées dans les équations (4) et (5). Le tableau IV présente les valeurs $Y_{p}$ et $Y_{b}$ pour les moments $t_{n}$, ainsi que les éclairements relatifs sous forêts $(\mathrm{Y} \%$ ) calculés pour la période d'une demi-longueur du jour.

Il résulte de ce tableau que l'éclairement relatif sous forêt diminue selon le passage du temps depuis le lever du soleil jusqu'au midi vrai. C'est-à-dire que le cours de l'éclairement relatif pendant le jour est fonction de la hauteur du soleil $\left(h_{0}\right):$ plus $h_{0}$ augmente, plus la valeur Y \% diminue. Pour une journée sereine et idéalisée il est évident que l'accroissement de la hauteur du soleil implique l'augmentation de l'éclairement absolu.

La courbe présentée dans la figure 3, résulte de la proportion des fonctions $\mathrm{Y}_{\mathrm{b}}(5)$ et $\mathrm{Y}_{\mathrm{p}}(4)$, et montre le cours de l'éclairement relatif pendant le jour choisi (le 9 septembre 1966).

Dans le développement en série de Fourier (1), $\frac{\mathrm{a}_{0}}{2}$ est une mesure pour l'intensité moyenne dans l'intervalle fermé. A l'aide des équations (4) et (5) il est ainsi possible de calculer l'éclairement relatif moyen sous forêt pendant la journée $(\overline{\mathrm{Y}} \%)$.

$$
\begin{aligned}
& a_{0} \text { dans } Y_{p}=108,436 \text { Klux } \\
& a_{0} \text { dans } Y_{b}=0,650 \text { Klux } \\
& \bar{Y} \%=0,599 \% .
\end{aligned}
$$

\section{TABLEAU IV}

Les valeurs de l'éclairement absolue en plein découvert $\left(Y_{\mathrm{p}}\right)$, sous foret $\left(Y_{n}\right)$ et l'éclairement relatif $(Y \%)$

correspondant aux moments successifs $t_{n}$ avec $\Delta t$ constant

\begin{tabular}{|c|r|r|l|}
\hline $\mathrm{t}_{\mathrm{n}}$ & $\mathrm{Y}_{\mathrm{p}}$ en Klux & $\mathrm{Y}_{\mathrm{b}}$ en Klux & $\mathrm{Y} \%$ \\
\hline $\mathrm{t}_{1}$ & $-1,228$ & 0,010 & \\
$\mathrm{t}_{2}$ & 1,875 & 0,037 & 1,973 \\
$\mathrm{t}_{9}$ & 6,563 & 0,075 & 1,142 \\
$\mathrm{t}_{4}$ & 12,833 & 0,121 & 0,942 \\
$\mathrm{t}_{5}$ & 20,595 & 0,172 & 0,835 \\
$\mathrm{t}_{6}$ & 29,649 & 0,226 & 0,762 \\
$\mathrm{t}_{7}$ & 39,692 & 0,279 & 0,702 \\
$\mathrm{t}_{8}$ & 50,299 & 0,331 & 0,658 \\
$\mathrm{t}_{9}$ & 60,977 & 0,380 & 0,623 \\
$\mathrm{t}_{10}$ & 71,242 & 0,424 & 0,595 \\
$\mathrm{t}_{11}$ & 80,642 & 0,464 & 0,575 \\
$\mathrm{t}_{12}$ & 88,829 & 0,499 & 0,561 \\
$\mathrm{t}_{13}$ & 95,603 & 0,529 & 0,553 \\
$\mathrm{t}_{14}$ & 100,885 & 0,554 & 0,549 \\
$\mathrm{t}_{15}$ & 104,705 & 0,573 & 0,547 \\
$\mathrm{t}_{16}$ & 107,163 & 0,586 & 0,546 \\
$\mathrm{t}_{17}$ & 108,361 & 0,593 & 0,546 \\
\hline
\end{tabular}




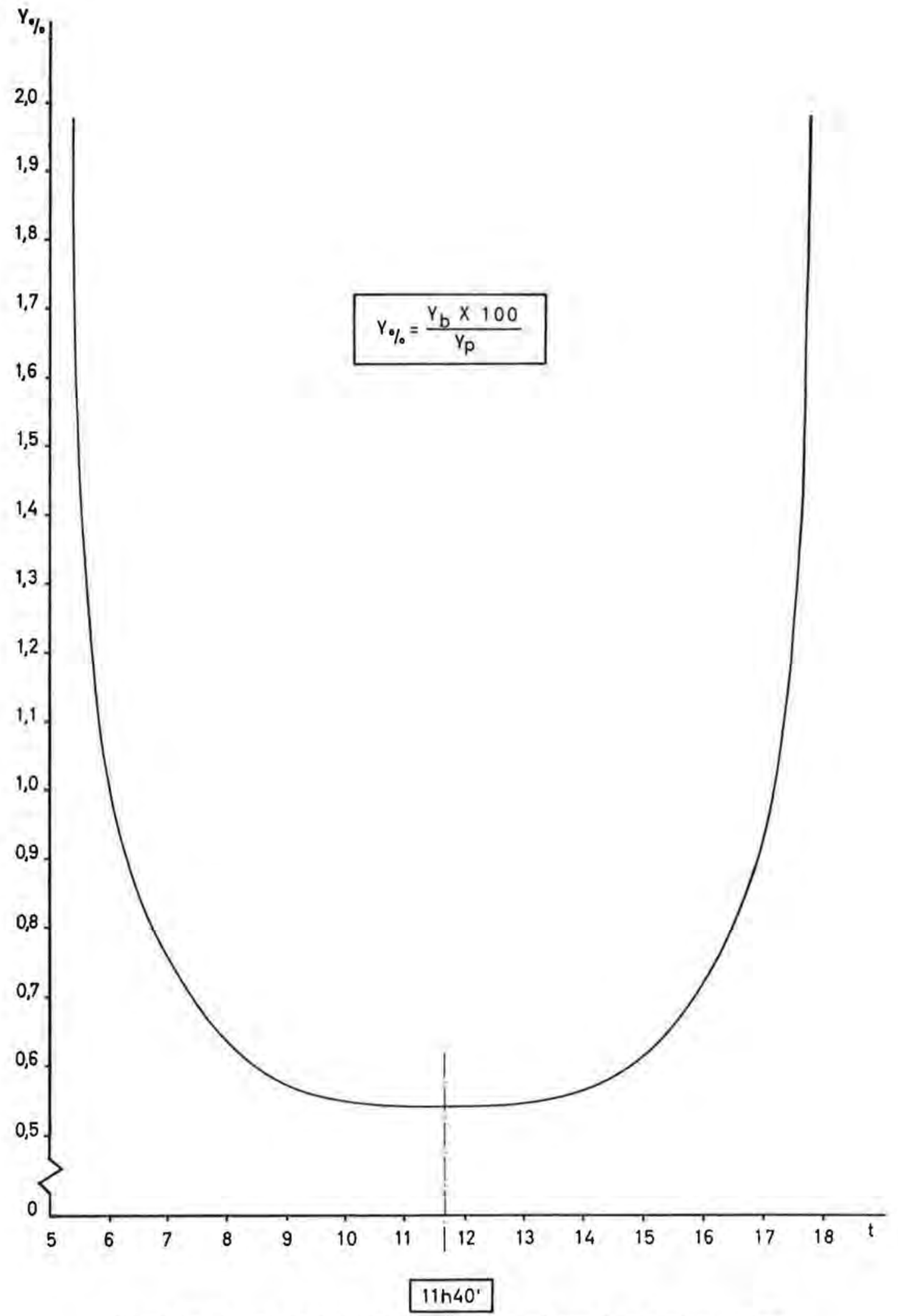

Figure 3: Le cours de l'éclairement relatif transmis sous forêt pendant un jour idéalisé du type de temps serein (le 9 septembre 1966). 
Ceci signifie que l'éclairement relatif moyen pour toute la journée $(\bar{Y} \%)$ est du même ordre de grandeur que les éclairements relatifs dans la période autour du midi vrai. Ensuite, il est évident que les valeurs pour des mesures instantanées de l'éclairement relatif, obtenues pendant la période de midi, sont plus représentatives que celles pour des mesures effectuées à d'autres moments.

Les mêmes considérations peuvent être faites en question du rapport entre l'éclairement relatif sous forêt et l'éclairement incident dans le cas d'un jour serein et idéalisé ou dans le cas des périodes d'observations. Pendant un jour serein le genre de lumière au midi vrai est surtout direct (les grandes intensités), par opposition à la lumière indirecte ou diffuse des périodes après le lever et avant le coucher du soleil. De nouveau, il est possible de conclure que la lumière directe est plus réfléchie par les couronnes que la lumière diffuse.

\section{Résumé et conclusions}

Dans la chênaie mélangée calcicole de la station écologique de Virelles (alt. $245 \mathrm{~m}$ ), le rapport entre l'éclairement relatif sous forêt et l'éclairement lumineux incident a été étudié,

1. Il n'était pas possible de trouver une relation mathématique en sortant des données hebdomadaires et journalières, fournies respectivement par des appareils totalisateurs et enregistreurs.

2. En travaillant avec les valeurs de l'éclairement au midi vrai, dérivées des diagrammes journaliers enregistrés pendant les périodes d'observation, il était possible de poser que l'éclairement relatif est fonction de l'éclairement absolu incident. L'éclairement relatif diminue avec l'augmentation de l'éclairement incident absolu.

3. Pour un changement identique de l'éclairement incident, la variation de la transmission relative devient plus petite selon que la fermeture du couvert feuillé se réalise ou que le niveau moyen d'ombrage devient plus petit.

4. Pour un jour serein et idéalisé l'éclairement relatif est étudié, L'éclairement relatif pendant la journée est fonction de la hauteur du soleil et donc en même temps de l'éclairement absolu incident.

5. Des mesures instantanées de l'éclairement relatif sous forêts pendant la période autour du midi ont une grande signification pour caractériser la valeur moyenne journalière de l'éclairement relatif.

6. La relation étudiée s'explique en posant que la lumière directe est plus réfléchie par le couvert feuillé que la lumière diffuse. 


\section{BIBLIOGRAPHIE}

BRECHTEL H.M. - Methodische Beiträge zur Oekologie der Ueberschirmung und Auflichtung einschichtige Waldbestände. Schriftenreihe der Landesfortsverwaltung Baden-Wirtlemberg B.14 1962.

BURSCHEL $P_{\text {, }}$ SGHMALTZ J, - Die Bedeutung des Lichtes für die Entwicklung junger Buchen, Allgem. Forst-u Jagdzeitung $136 \mathrm{Jg}$. H9 1965.

GEIGER R. - Das Klima der bodennahen Luftschicht. Verlag Vieweg II Auf. 1961

GRULOIS J. \& VYNCKE G. - Comparaison entre les éclairements lumineux et encrgćtique incidents et transmis sous forêt en phénophase feuilléc. Oecologia plantarum - Montpellier 1969 (inédit).

MITSCHERLICH et al. - Ertragskundlich-ökologische Untersuchungen im Reien- und Mischbestand. Allgem. Forst-u. Jagdzeilung 136 Jg. H. 10-12 $1965 ; 137 \mathrm{Jg} . \mathrm{H} 1-51966$.

SAUBERER F, \& HAERTEL O. - Pflanze und Strahlung. Akadem. Verlagsges. Geest \& Porlig K, - G. Leipzig 1959.

VAN MIEGROET M. \& SEGERS M. - Recherches sur l'intensité et la qualité de la lumière en forêt. Lejeunia Liège. Nouv. Série n $1^{\circ} 24$ octobre 1963.

VAN MIEGROET M. - Die Lichttransgression und die Lichtreflexion bei Blättern einiger Laubbaumarten. Schweizerische Zeitschrift filr Forstwesen No. 71965.

VYNCKE G. \& GOOSSENS R. - An apparatus for the instantaneous measurement of the relative light intensity. Sylva Gandavensis No. 111968.

\section{SAMENVATTING}

Het verband tussen de relatieve verlichtingssterkte in het bos en de absolute invallende verlichtingssterkte tijdens de groeiperiode

In het ekologisch station van Virelles (h $235 \mathrm{~m}$ ) werd het verband bestudeerd tussen de relatieve verlichtingssterkte in een gemengd eikenbos op kalkgrond en de absolute verlichtingssterkte boven het scherm.

1. Uitgaande van de wekelijkse en dagelijkse verlichtingssterkten, bekomen door metingen respektievelijk met sommerende en registrerende apparatuur, kon geen wiskundig verband worden vastgesteld.

2. De verlichtingssterkte op de ware middag, afgeleid uit dagelijkse geregistreerde kurven, lieten toe cen verband vast te stellen voor elke onderzoeksperiode.

De relatieve verlichtingssterkte is een funktie van de absolute verlichtingssterkte boven het scherm, deze vermindert met toenemende absolute verlichtingssterkte.

3. De variatic van de relaticve verlichtingssterkte voor een identische verandering van de absolute verlichtingssterkte boven het scherm, wordt kleiner naarmate de kroonsluiting zich yoltrekt of naargelang het gemiddeld schaduwniveau kleiner wordt.

4. Tijdens cen geidealisecrde dag van het klaarweertype is de relatieve verlichtingssterkte funktie van de zonnehoogte en dus terzelfdertijd van de absolute verlichtingssterkte boven het scherm.

5. Een goede maat voor de gemiddelde dagelijkse relatieve verlichtingssterkte kunnen deze relatieve verlichtingssterkte geven welke rechtstreeks gemeten worden in de periode rond de ware middag.

6. De bestudeerde relatie kan verklaard worden door het feit dat direkt licht meer wecrkaatst wordt door het bladerenscherm dan diffuus licht. 


\section{SUMMARY}

The relation between the relative light intensity in a forest stand and the absolute light intensity above the canopy during the growing period

In the research center of Virelles (mixed oak forest) the relation between the relative light intensity and the absolute incident light intensity was studied. Only for the light intensity values measured on the real noon, a mathematical relation was found and this for the growing periods 1965 and 1966. With the decrease of the light intensity in the open, the relative light intensity inc reases.

For an identical change of the incident light intensity, the variation of the relative transmission becomes smaller, according as the crown contact becomes more intense.

The relative light intensity under the canopy, during an ideal day of the clear weather type, is a function of the sun-height and at the same time of the absolute light intensity in the open.

A measure for the mean relative light intensity of some day, can be given by instantaneous measurements of the relative light intensity, only during the real noon period.

The found relation may be explained by the fact that direct incident light is more reflected by the canopy than diffused light. 